5. E. C. Titchmarsh, The Theory of Functions, Oxford Univ. Press, London, 1939.

6. E. C. Titchmarsh, Eigenfunction Expansions Associated With Second-Order Differential Equations, Oxford, at the Clarendon Press, 1946; Part I, Oxford Univ. Press, London, 1962. MR 8, 458.

7. G. N. Watson, A Treatise on the Theory of Bessel Functions, 2nd ed., Cambridge Univ. Press and Macmillan, New York, 1944. MR 6, 64.

8. W. H. Young, "On series of Bessel functions," Proc. London Math. Soc., (2), v. 18, 1920, pp. 163-200.

\title{
On a Numerical Solution of an Integral Equation with Singularities
}

\author{
By Robert G. Voigt
}

1. Introduction. Annular airfoil theory gives rise to Fredholm integral equations of the second kind in the following form:

$$
f(x)=g(x)+\int_{0}^{1} G(x, y) f(y) d y, \quad 0 \leqq x \leqq 1,
$$

where the kernel $G(x, y)$ has the form

$$
G(x, y)=\int_{0}^{1} \frac{q(y, z)}{z-x} d z
$$

and $g(x)$ is a continuous function; in particular it may be of the form

$$
g(x)=\int_{0}^{1} \frac{r(x, z)}{z-x} d z .
$$

For what follows, we will assume that $q(y, z)$ and $r(x, z)$ are continuous functions as they would be in most physical problems; however, the results are valid for more general functions. By using a Fourier series technique given in Collatz [1], we are able to neatly evaluate the singular integrals involved, but as will be seen, this is not the only advantage of the technique. We also obtain a kernel function of degenerate type; that is

$$
G(x, y)=\sum_{i=1}^{n} m_{i}(x) M_{i}(y)
$$

Then the integral equation may be solved using a method applicable to degenerate kernels such as the simple one given in Mikhlin [2].

An example of the method applied to an integral equation arising in annular airfoil theory is included at the end of this paper.

2. Handling the Singularities. The first step in handling the singularities is to apply the changes of variables suggested by Collatz [1]: Let

$$
\begin{aligned}
& x=\frac{1}{2}(1+\cos \theta), \\
& y=\frac{1}{2}(1+\cos \psi), \\
& z=\frac{1}{2}(1+\cos \varphi) .
\end{aligned}
$$

Received April 22, 1965. 
Equation (1) may then be written in the form

$$
f^{*}(\theta)=g^{*}(\theta)+\frac{1}{2} \int_{0}^{\pi} G^{*}(\theta, \psi) f^{*}(\psi) \sin \psi d \psi,
$$

where

$$
G^{*}(\theta, \psi)=\int_{0}^{\pi} \frac{q^{*}(\psi, \varphi) \sin \varphi}{\cos \varphi-\cos \theta} d \varphi
$$

The ${ }^{*}$ is used to simplify notation, e.g.,

$$
f(x)=f\left[\frac{1}{2}(1+\cos \theta)\right] \equiv f^{*}(\theta) .
$$

Now we may expand $q^{*}(\psi, \varphi) \sin \varphi$ in a Fourier cosine series obtaining

$$
G^{*}(\theta, \psi)=\int_{0}^{\pi} \frac{\sum_{n=0}^{\infty} a_{n}(\psi) \cos n \varphi}{\cos \varphi-\cos \theta} d \varphi
$$

Since $q^{*}(\psi, \varphi) \sin \varphi$ is a continuous function of $\varphi$, the series is uniformly convergent. Thus we may write

$$
G^{*}(\theta, \psi)=\sum_{n=0}^{\infty} a_{n}(\psi) \int_{0}^{x} \frac{\cos n \varphi}{\cos \varphi-\cos \theta} d \varphi
$$

If we now consider the Cauchy Principal Value of the integral, we have that

$$
G^{*}(\theta, \psi)=\pi \sum_{n=1}^{\infty} \frac{a_{n}(\psi) \sin n \theta}{\sin \theta}
$$

Since the infinite sum is convergent, we may approximate it to a prescribed degree of accuracy with a finite sum. This yields a kernel of degenerate type.

If $g(x)$ is given by Equation (2) it may be evaluated in the same way that $G(x, y)$ was evaluated. Thus we obtain Equation (1) in a form free of singularities.

3. Solution of the Equation. Equation (1) now has the form

$$
f^{*}(\theta) \doteq g^{*}(\theta)+\frac{\pi}{2} \int_{0}^{\pi} \sum_{n=1}^{N} \frac{a_{n}(\psi) \sin n \theta}{\sin \theta}\left[\sin \psi f^{*}(\psi)\right] d \psi
$$

Proceeding with the method of solution found in Mikhlin [2] we write Equation (3) in the form

$$
f^{*}(\theta) \doteq g^{*}(\theta)+\frac{\pi}{2} \sum_{n=1}^{N} \frac{\sin n \theta}{\sin \theta} C_{n}
$$

where

$$
C_{n}=\int_{0}^{\pi} a_{n}(\psi) \sin \psi f^{*}(\psi) d \psi
$$

Using Equation (4) in the expression for $C_{n}$ we may write

$$
C_{n}-\int_{0}^{\pi} a_{n}(\psi) \sin \psi\left[g^{*}(\psi)+\frac{\pi}{2} \sum_{m=1}^{N} \frac{\sin m \psi}{\sin \psi} C_{m}\right] d \psi \doteq 0
$$


Since this is true for $n=1,2, \cdots, N$, we have the following linear system:

$$
C_{n}-\frac{\pi}{2} \sum_{m=1}^{N} \alpha_{n, m} C_{m} \doteq \beta_{n}, \quad n=1,2, \cdots, N,
$$

where

$$
\beta_{n}=\int_{0}^{\pi} a_{n}(\psi) \sin \psi g^{*}(\psi) d \psi,
$$

and

$$
\alpha_{n, m}=\int_{0}^{\pi} a_{n}(\psi) \sin m \psi d \psi .
$$

After evaluating $\beta_{n}$ and $\alpha_{n, m}$, the system (5) may be solved for $C_{n}$. Substitution of $C_{n}$ values into Equation (4) then gives a solution to (1).

4. Example. The following equation arises as part of the solution of the problem of finding the shape of an annular airfoil from a specified pressure distribution on the inner and outer surfaces of the airfoil, $p^{-}(x)$ and $p^{+}(x)$, respectively [3]. The annular airfoil has been nondimensionalized to have length 1 with the trailing edge at 0 , thus $x \in[0,1]$. The equation is in the form of Equation (1) with $g(x)$ in the form of Equation (2):

$$
\begin{aligned}
f(x) & =\frac{1}{\pi^{2} \sqrt{ }(x(1-x))}\left\{-\int_{0}^{1} \frac{r(x, z)}{z-x} d z+\int_{0}^{1}\left[\int_{0}^{1} \frac{q(y, z)}{z-x} d z\right] f(y) d y\right\}, \\
r(x, z) & =\sqrt{ }(z(1-z))\left\{\frac{h}{2} \int_{0}^{1} u(t) k(z, t)[K[k(z, t)]-E[k(z, t)]] d t+\pi w(z)\right\}, \\
q(y, z) & =\frac{\sqrt{ }(z(1-z))}{z-y}\{1-k(y, z) E[k(y, z)]\} .
\end{aligned}
$$

The apparent square root singularities at $x=0$ and $x=1$ are removed when the changes of variables are made since $f(x)$ represents a first derivative; i.e., the slope of the thickness distribution. Thus for this particular example

$$
f(x)=-\frac{f^{*}(\theta) \sin \theta}{2} .
$$

$K[k(z, t)]$ and $E[k(z, t)]$ are complete elliptic integrals of the first and second kind, respectively, with modulus $k(z, t)=1 / \sqrt{ }\left(h^{2}(z-t)^{2}+1\right)$. The quantity $h$ is a specified constant, and $u(t)$ and $w(z)$, which are given, depend on $p^{-}$and $p^{+}$; a complete discussion is given in Reference [3].

The above example has been run on the IBM-7090 at the David Taylor Model Basin. The following table illustrates the effect of the number of coefficients on the stability of the solution. $N$ is the number of coefficients used in the evaluation of the kernel function $G$; the next column is the number of significant places in the integral term of Equation (1) unaffected by increasing $N ; M$ is the number of co- 
efficients used in the evaluation of $g$; and the last column is the number of significant places in $g$ unaffected by increasing $M$.

\begin{tabular}{|c|c|c|c|}
\hline$N$ & & $M$ & \\
\hline $\begin{array}{r}5 \\
10 \\
15 \\
20 \\
25 \\
30 \\
35 \\
40\end{array}$ & $\begin{array}{l}4 \\
5 \\
5 \\
6 \\
6 \\
6 \\
6 \\
6\end{array}$ & $\begin{array}{r}5 \\
10 \\
15 \\
20 \\
25 \\
30 \\
35 \\
40 \\
45 \\
50 \\
55\end{array}$ & $\begin{array}{l}2 \\
2 \\
2 \\
2 \\
2 \\
2 \\
3 \\
3 \\
4 \\
4 \\
4\end{array}$ \\
\hline
\end{tabular}

This table indicates that a better method should be investigated for the evaluation of $g$; however, in evaluating the kernel, the Fourier series technique seems to be satisfactory, and it is in this part of the problem that the two-fold advantage of the technique is realized. The present program requires less than two minutes on the 7090 for $N=20$ and $M=45$.

5. Acknowledgment. This paper covers research initiated by the Hydromechanics Laboratory, David W. Taylor Model Basin, Washington, D. C., under Subproject No. S-R011-0101, Task 0401.

David Taylor Model Basin

Hydromechanics Laboratory

Washington, D. C.

1. L. Coluatz, The Numerical Treatment of Differential Equations, 3rd ed. of English transl., Die Grundlehren der Mathematischen Wissenschaften, Bd. 60, Springer, Berlin, 1960, pp. 505-506. MR 22 *322.

2. S. G. Miknlin, Integral Equations, Macmillan, New York, 1957, pp. 19-21.

3. W. B. Morgan, "Theory of the annular airfoil and ducted propeller," Fourth Symposium on Naval Hydrodynamics, ACR-92, U. S. Government Printing Office, Washington, D. C., 1962, pp. 151-197. 